\title{
Ambulatory electrocardiogram analysis in infants treated with recombinant human acid $\alpha$-glucosidase enzyme replacement therapy for Pompe disease
}

\author{
Amanda L. Cook, $M D^{1}$, Priya S. Kishnani, $M D^{2}$, Michael P. Carboni, $M D^{1}$, Ronald J. Kanter, $M D^{1}$, \\ Y. T. Chen, MD, PhD' ${ }^{2}$, Annette K. Ansong, $M D^{1}$, Richard M. Kravitz, $M D^{3}$, Henry Rice, $M D^{4}$, and Jennifer S. Li, $M D^{1}$
}

\begin{abstract}
Purpose: Infantile Pompe disease is caused by deficiency of lysosomal acid $\alpha$-glucosidase. Trials with recombinant human acid $\alpha$-glucosidase enzyme replacement therapy (ERT) show a decrease in left ventricular mass and improved function. We evaluated 24-hour ambulatory electrocardiograms (ECGs) at baseline and during ERT in patients with infantile Pompe disease. Methods: Thirty-two ambulatory ECGs were evaluated for 12 patients with infantile Pompe disease from 2003 to 2005. Patients had a median age of 7.4 months (2.9-37.8 months) at initiation of ERT. Ambulatory ECGs were obtained at determined intervals and analyzed. Results: Significant ectopy was present in 2 of 12 patients. Patient 1 had 211 and 229 premature ventricular contractions $(0.2 \%$ of heart beats) at baseline and at 11.5 weeks of ERT, respectively. Patient 2 had 10,445 premature ventricular contractions (6.7\% of heart beats) at 11 weeks of therapy. Conclusion: Infantile Pompe disease may have preexisting ectopy; it may also develop during the course of ERT. Therefore, routinely monitoring patients using 24-hour ambulatory ECGs is useful. Periods of highest risk may be early in the course of ERT when there is a substantial decrease in left ventricular mass and an initial decrease in ejection fraction. Genet Med 2006:8(5):313-317.
\end{abstract}

Key Words: glycogen storage disease type II, Pompe disease, acid maltase deficiency, enzyme replacement therapy, cardiomyopathy, arrhythmias, ventricular ectopy

Pompe disease or glycogen storage disease type II is an autosomal recessive disorder with an incidence of 1 in 40,000 live births. ${ }^{1}$ It is secondary to lysosomal deficiency of acid $\alpha$-glucosidase (GAA) resulting in the excessive accumulation of glycogen within the lysosomes of many cells including smooth, skeletal, and cardiac muscle cells. There is a spectrum of disease severity depending on the amount of residual enzyme activity. At the most severe end of the disease spectrum is infantile Pompe disease with residual enzyme activity of less than $1 \%$ of normal. ${ }^{2}$ These patients typically present within the first few months of life with hypotonia, hypertrophic cardiomyopathy,

\footnotetext{
From the Divisions of ${ }^{1}$ Pediatric Cardiology, ${ }^{2}$ Genetics, ${ }^{3}$ Pulmonary, and ${ }^{4}$ Surgery, Duke University Medical Center, Durham, North Carolina.

Jennifer S. Li, MD, Division of Pediatric Cardiology, Duke University Medical Center, Box 3090, Durham, NC 27710.

Submitted for publication December 5, 2005.

Accepted for publication February 14, 2006.

Source of Funding and Conflict of Interest Statements: The enzyme replacement therapy trials are supported by a grant from Genzyme Corporation and by M01-RR30, National Center for Research Sources, General Clinical Research Centers Program. P.S.K. and Y.T.C. received research/grant support from Genzyme Corporation; P.S.K. is a member of the Pompe Disease Registry Advisory Board for Genzyme Corporation. Y.T.C. served as a consultant for Genzyme Corporation. If therapy for Pompe disease proves successful commercially, Duke University and inventors for the cell line used to generate the enzyme used in this clinical trial may benefit financially pursuant to the University's Policy on Inventions, Patents and Technology Transfer.
}

DOI: 10.1097/01.gim.0000217786.79173.a8 hepatomegaly, and frequent respiratory infections. Without treatment, infantile Pompe disease is typically fatal before 1 year of age. ${ }^{3}$ Signs and symptoms in those with late-onset (juvenile and adult-onset) Pompe disease are more variable, but typically include progressive muscular weakness and respiratory problems with few developing cardiac manifestations. ${ }^{2}$

Patients with infantile Pompe disease develop a cardiomyopathy secondary to the massive accumulation of glycogen within the cardiac muscle. They have biventricular hypertrophy including hypertrophy of the interventricular septum, which can be present in some instances at birth and in others within the first year of life. Hypertrophy may result in left ventricular outflow tract obstruction and in the later stages progresses to a dilated cardiomyopathy. In a natural history study of infantile Pompe disease, heart failure was noted by age 4.5 months. ${ }^{3}$ Glycogen accumulation also affects the specialized conduction tissue cells and gross depolarization and repolarization patterns, resulting in characteristic electrocardiographic changes, including shortened PR interval, increased QT dispersion, and large QRS complexes. ${ }^{4}$ The shortened PR interval may on occasion be the result of true Wolff-Parkinson-White syndrome, placing these patients at risk for supraventricular tachyarrhythmias. ${ }^{5,6}$ In our review of the literature, there have not been case reports of ventricular ectopy. However, the hypertrophic cardiomyopathy along with conduction system abnormalities theoretically would place these patients at risk for ventricular arrhythmias and sudden death. ${ }^{5-7}$ 
With enzyme replacement therapy (ERT) of recombinant human GAA, clinical trials have demonstrated a remarkable decrease in the left ventricular mass index and normalization of ventricular function as early as after 12 weeks of ERT. ${ }^{8-12}$ Conduction abnormalities in patients with infantile Pompe disease may also improve after ERT, with an increase in PR interval and decrease in both QT dispersion and left ventricular voltage. ${ }^{13}$ It is our hypothesis that although ERT results in improvement of cardiomegaly and function, patients with infantile Pompe disease may still be at risk for arrhythmias secondary to glycogen clearance and remodeling of the conduction system.

\section{METHODS}

Twelve patients ( 4 female, 8 male) with infantile Pompe disease with a median age of 7.4 months (2.9-37.8 months) at the start of ERT were enrolled in open-label clinical trials exploring the safety and efficacy of Chinese hamster ovary cellderived recombinant human GAA ERT at Duke University Medical Center from 2003 to 2005. All patients had a confirmed diagnosis of Pompe disease with an endogenous GAA enzyme activity of less than $1 \%$ of the normal mean in skin fibroblasts. GAA enzyme activity was assessed by measurement of 4-methyl-umbelliferyl- $\alpha$-D-glucoside cleavage at $\mathrm{pH} 4.3$. All patients had cardiomegaly (cardiothoracic ratio $>0.5$ by chest $\mathrm{x}$-ray film) and cardiac hypertrophy (left ventricular mass index $>65 \mathrm{~g} / \mathrm{m}^{2}$, equivalent to 2 or more standard deviations above the normal mean). ${ }^{14}$ The doses of Chinese hamster ovary cell-derived recombinant human GAA enzyme ranged from 20 to $40 \mathrm{mg} / \mathrm{kg}$ every 2 weeks.

Ambulatory electrocardiograms (ECGs) were performed at baseline and repeated at approximately 3 months, 6 months, 1 year, and as indicated. These studies were approved by the Duke Institutional Review Board with written informed consent obtained from the patient's guardian. Twenty-four hour ambulatory ECGs were obtained using one of two different monitors: Zymed DigiTrak-Plus 3100A (Philips Medical, Andover, Mass) and Zymed MultiTrak 1100-010 (Philips Medical). After collection, the data were downloaded and scanned with a Philips Zymed 2010 scanner. All beats were reviewed with analysis of the following data: average, maximum, and minimum heart rates; R-R intervals; QRS morphology; and premature atrial and ventricular contractions. Premature atrial contractions were electronically diagnosed on the basis of an $\mathrm{R}-\mathrm{R}$ interval that was $25 \%$ shorter than the previous $\mathrm{R}-\mathrm{R}$ interval associated with a preceding $\mathrm{P}$ wave and overread as not representing marked sinus arrhythmia or sinoatrial conduction block. Premature ventricular contractions were electronically diagnosed on the basis of a QRS morphology different than the sinus QRS template and overread to confirm prematurity and to rule out supraventricular beats with bundle branch aberrancy. A pediatric electrophysiologist reviewed the data and confirmed the number of premature ventricular and atrial contractions.
Echocardiograms were obtained on all 12 patients at baseline and at 4, 8, 12, 24, 36, and 52 weeks of ERT. Echocardiographic evaluation consisted of a complete two-dimensional cross sectional, $\mathrm{M}$-mode, and Doppler examination using a Siemens Sequoia system (Siemens Medical Solution, Mt. View, Calif) or a Philips 5500 or 7500 system (Philips Medical Systems North America Company, Bothell, Wash). Left ventricular mass was measured by the area-length method using an average of three to five independent measurements from the same study. ${ }^{15}$ The ejection fraction was calculated on the basis of the modified Simpson's rule formula. ${ }^{16}$

\section{RESULTS}

A total of 12 patients were enrolled ( 8 male and 4 female) with infantile Pompe disease and a median age of 7.4 months (2.9-37.8 months) at the start of ERT. They received ERT for 19 to 139 weeks (median 100 weeks). Changes in the ejection fraction and left ventricular mass are shown in Figures 1 and 2. A total of 80 echocardiograms were performed on the 12 patients. The left ventricular mass at baseline ranged from 179 to $404 \mathrm{~g} / \mathrm{m}^{2}$ (median $269 \mathrm{~g} / \mathrm{m}^{2}$ ). After 10 to 12 weeks of ERT the left ventricular mass decreased to a range of 115 to $305 \mathrm{~g} / \mathrm{m}^{2}$

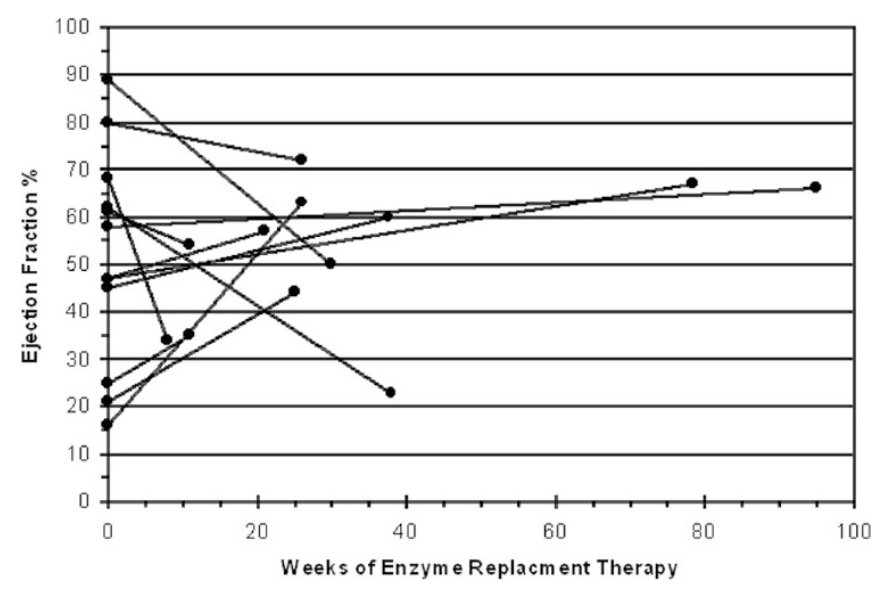

Fig. 1 Ejection fraction changes of the 12 patients during enzyme replacement therapy (ERT).

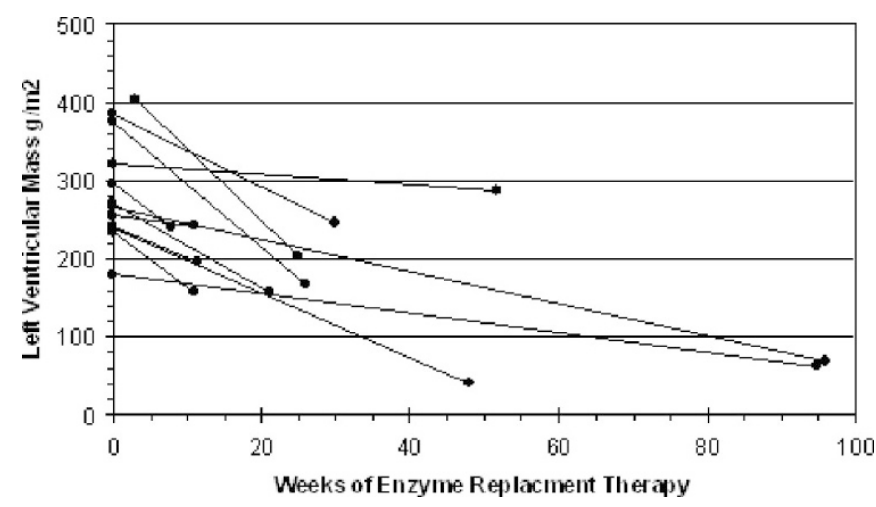

Fig. 2 Decrease in left ventricular mass in all 12 patients during weeks of enzyme replacement therapy (ERT). 
(median $242 \mathrm{~g} / \mathrm{m}^{2}$ ). There was a significant reduction in left ventricular mass during ERT (Fig. 1) with a range of 40 to 286 $\mathrm{g} / \mathrm{m}^{2}$ (median $180 \mathrm{~g} / \mathrm{m}^{2} ; P<.001$ ). The ejection fraction at baseline ranged from $16 \%$ to $89 \%$ (median $58 \%$ ) and tended to decrease for the first several weeks after ERT. It then tended to normalize after several more weeks of ERT (10-15 weeks) (Fig. 2).

Table 1

Ambulatory electrocardiogram findings

\begin{tabular}{|c|c|c|c|c|c|c|}
\hline Patient & $\begin{array}{l}\text { Weeks } \\
\text { of ERT }\end{array}$ & PAC & PVC & $\begin{array}{c}\text { Min } \\
\mathrm{HR} / \mathrm{min}\end{array}$ & $\begin{array}{c}\text { Avg } \\
\mathrm{HR} / \mathrm{min}\end{array}$ & $\begin{array}{c}\text { Max } \\
\mathrm{HR} / \min \end{array}$ \\
\hline \multirow[t]{2}{*}{1} & 0 & $1(0)$ & $211(0.2)$ & 55 & 87 & 145 \\
\hline & 11.5 & 0 & $229(0.2)$ & 47 & 77 & 152 \\
\hline \multirow[t]{7}{*}{2} & 0 & 0 & 0 & 83 & 119 & 162 \\
\hline & 11 & 0 & $10,445(6.7)$ & 83 & 119 & 182 \\
\hline & 12 & 0 & $2565(1.8)$ & 88 & 136 & 222 \\
\hline & 13 & 0 & $1585(1.8)$ & 70 & 116 & 174 \\
\hline & 36 & $1(0)$ & 0 & 81 & 128 & 179 \\
\hline & 51.5 & 0 & $14(0)$ & 101 & 134 & 179 \\
\hline & 78 & $5(0)$ & $42(0)$ & 71 & 115 & 190 \\
\hline \multirow[t]{3}{*}{3} & 1 & 0 & $1(0)$ & 83 & 132 & 174 \\
\hline & 13 & $8(0)$ & 0 & 75 & 116 & 162 \\
\hline & 27 & 0 & 0 & 76 & 129 & 171 \\
\hline \multirow[t]{4}{*}{4} & 0 & $5(0)$ & $1(0)$ & 69 & 119 & 169 \\
\hline & 6 & $4(0)$ & $3(0)$ & 63 & 107 & 171 \\
\hline & 27 & $5(0)$ & 0 & 69 & 114 & 160 \\
\hline & 39.5 & $1(0)$ & 0 & 63 & 110 & 169 \\
\hline \multirow[t]{5}{*}{5} & 0 & $38(0)$ & $1(0)$ & 76 & 150 & 214 \\
\hline & 9 & $1(0)$ & 0 & 77 & 144 & 188 \\
\hline & 11 & $1(0)$ & $44(0)$ & 62 & 145 & 182 \\
\hline & 12.5 & $12(0)$ & 0 & 58 & 128 & 190 \\
\hline & 12.6 & $9(0)$ & $4(0)$ & 63 & 136 & 176 \\
\hline 6 & 27 & 0 & 0 & 73 & 120 & 185 \\
\hline \multirow[t]{2}{*}{7} & 0 & $1(0)$ & $1(0)$ & 81 & 125 & 190 \\
\hline & 53 & $5(0)$ & 0 & 70 & 114 & 179 \\
\hline 8 & 13 & $41(0)$ & 0 & 85 & 127 & 211 \\
\hline \multirow[t]{3}{*}{9} & 0 & $19(0)$ & $9(0)$ & 69 & 122 & 188 \\
\hline & 10 & 0 & 0 & 76 & 123 & 171 \\
\hline & 28 & 0 & $2(0)$ & 107 & 145 & 190 \\
\hline \multirow[t]{2}{*}{10} & 0 & $8(0)$ & $8(0)$ & 68 & 105 & 148 \\
\hline & 11 & 0 & 0 & 63 & 108 & 197 \\
\hline 11 & 0 & $2(0)$ & 0 & 81 & 121 & 182 \\
\hline 12 & 0 & $44(0)$ & 0 & 77 & 116 & 188 \\
\hline
\end{tabular}

Thirty-three ambulatory electrocardiograms (ECGs) of the 12 patients during enzyme replacement therapy. PAC, premature atrial contractions; PVC, premature ventricular contractions; Min HR, minimum heart rate; Avg HR, average heart rate; Max HR, maximum heart rate.
A total of 32 ambulatory ECGs were obtained from 12 patients. Ten of 12 patients underwent 24-hour ambulatory ECG analysis at baseline before initiating ERT. The other two patients underwent the initial ambulatory ECG analysis at 1 week and 13 weeks after starting ERT. Each patient had between one and seven ambulatory ECG studies. The ambulatory ECGs were evaluated for R-R intervals and minimum, average, and maximum heart rates. They were also evaluated for supraventricular and ventricular ectopy as well as for other arrhythmias. The R-R intervals ranged from 0.9 to 1.4 seconds (median 0.9 seconds). The maximum heart rates ranged from 152 to 211 beats/min (median 182 beats/min); average heart rates ranged from 77 to 128 beats/min (median 119 beats/min); and minimum heart rates ranged from 47 to 88 beats/min (median 63 beats/min) (Table 1).

Two of 12 patients were noted to have premature ventricular contractions on the ambulatory ECG. Patient 1 was noted to have $0.2 \%$ premature ventricular contractions on an ambulatory ECG at baseline and at 11.5 weeks. Patient 1 initially had a left ventricular mass of $241 \mathrm{~g} / \mathrm{m}^{2}$ and an ejection fraction of $80 \%$. At 11.5 weeks of ERT the left ventricular mass decreased to $195 \mathrm{~g} / \mathrm{m}^{2}$ and the ejection fraction was $72 \%$. Patient 2 was noted to have a peak percentile of premature ventricular contractions during week 11 of ERT (Fig. 3). The patient was started on mexiletine with resolution of the ectopic ventricular beats. Patient 2 initially had a left ventricular mass of $179 \mathrm{~g} / \mathrm{m}^{2}$, which decreased to $142 \mathrm{~g} / \mathrm{m}^{2}$ at 11 weeks of ERT and then further decreased to $72 \mathrm{~g} / \mathrm{m}^{2}$ by the most recent echocardiogram at 78 weeks of ERT (Fig. 4). The timing of the onset of

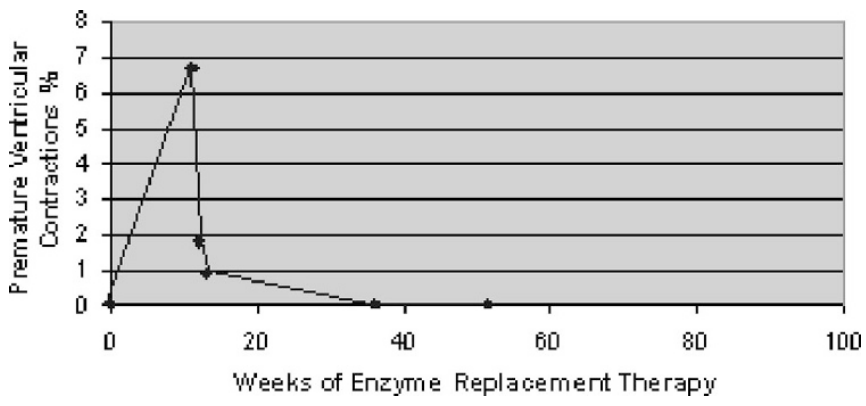

Fig. 3 Percentile of premature ventricular contractions in patient 2 during weeks of enzyme replacement therapy (ERT).

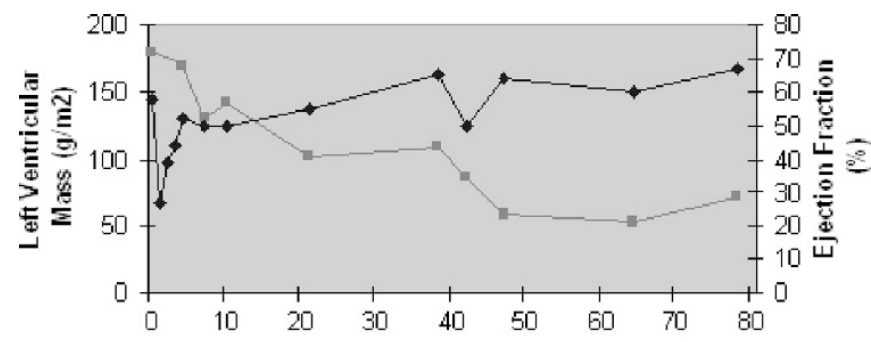

Weeks of Enzyme Replacement Therapy

$$
\text { - - Left Ventricular Mass }(\mathrm{g} / \mathrm{m} 2) \rightarrow \text { Ejection Fraction }
$$

Fig. 4 Changes in left ventricular mass and ejection fraction in patient 2 during weeks of enzyme replacement therapy (ERT). Time of peak (arrow). 
premature ventricular contractions correlated with the most rapid decrease in left ventricular mass. One of the 12 patients was noted to have Wolff-Parkinson-White changes on the ECG; however, this patient did not have ectopy on the ambulatory ECG. Patient 3 was noted to have multiple premature ventricular contractions on 12 lead ECG before starting ERT; however, this was one of the two patients who did not receive a baseline ambulatory ECG. Patient 3's first ambulatory ECG was obtained after 1 week of ERT and did not demonstrate any ectopy.

\section{DISCUSSION}

The natural history of infantile Pompe disease without treatment is poor. Affected infants present with hypertrophic cardiomyopathy, hypotonia, and generalized muscle weakness in the first few months of life followed by death from cardiorespiratory failure usually by 1 year. ${ }^{3,17}$ Most patients with infantile Pompe disease (95\%) develop progressive cardiomegaly before 6 months of age secondary to glycogen accumulation within the myocardium. ${ }^{4}$ Cardiomegaly has been diagnosed immediately after delivery, indicating that the hypertrophy may begin in utero. ${ }^{17}$ The cardiomegaly is characteristically massive and rapidly progressive resulting in both systolic and diastolic dysfunction, which may progress to a dilated cardiomyopathy further worsening cardiac output. The interventricular hypertrophy may result in left ventricular outflow tract obstruction.

The glycogen deposition also affects the specialized conduction tissue resulting in classic ECG findings: shortened PR interval, large QRS complexes, and increased QT dispersion. Several patients have also been reported to have Wolff-Parkinson-White syndrome., ${ }^{5,6}$ Wolff-Parkinson-White syndrome is typically secondary to a muscular tract crossing through the annulus fibrosis. In glycogen storage diseases the annulus fibrosis is disrupted by glycogen-filled myocytes rather than a distinct bypass tract. ${ }^{18}$

Results from several trials of recombinant human GAA in infantile-onset Pompe disease have demonstrated sustained improvement in the cardiomyopathy of all patients as evaluated by left ventricular mass index and left ventricular posterior wall thickness regardless of disease severity at onset of treatment. In these trials, improvements in cardiac function has also been noted. ${ }^{8-12}$ In addition, there is substantial improvement in the ECG after ERT including an increase in PR interval, a decrease in left ventricular voltage, and a shortening of QT dispersion. It is postulated that the reduction of glycogen within the conduction system and myocardium results in these changes. ${ }^{13}$ Patients with Fabry disease treated with ERT have also demonstrated improvement in left ventricular mass and ejection fraction with one study showing a patient developing persistent atrial fibrillation. ${ }^{19}$

Despite the significant improvement in conduction and the cardiomyopathy, patients likely continue to have abnormalities placing them at risk for arrhythmias that could be lethal. Twelve weeks into ERT is the average time for the most rapid decrease in left ventricular mass in our 12 patients (Fig. 2). Initially there is a decrease in the ejection fraction after initiation of the ERT, followed by improvement (Fig. 1). Patient 1 had premature ventricular contractions at baseline and at 11.5 weeks of ERT, which did not correlate with a rapid decrease in left ventricular mass or a change in ejection fraction. Patient 2 did not have any significant decrease in the ejection fraction at the time of the most frequent premature ventricular contractions; however, he did have a rapid decrease in left ventricular mass at this time (Fig. 4). This decrease in left ventricular mass occurred at the time of maximal ventricular ectopy at 11 weeks of ERT. Further investigation is indicated to more completely evaluate the possible correlation between rapid left ventricular mass reduction, decreasing ejection fraction, and ventricular ectopy.

Although unknown, remodeling of the myocardium may develop secondary to glycogen clearance from the myocytes resulting in fibrous tissue deposition. The correlation of increased ventricular ectopy seen in 1 patient of 12 during the time of rapid decrease in left ventricular mass suggests remodeling during ERT as a possible cause. Fibrous tissue deposition during the clearance of glycogen from the myocytes may place these patients at risk for ventricular ectopy including ventricular tachycardia. In addition, premature ventricular contractions in a patient with Wolff-Parkinson-White syndrome may be a trigger for supraventricular tachycardia. These potentially lethal arrhythmias may also be present at baseline (such as in patient 1) and may not improve initially with ERT.

Our study was limited by the small number of patients enrolled. However, because 1 of 12 patients developed ventricular ectopy during ERT, we believe that further screening in patients receiving ERT is warranted. Efforts should continue to monitor patients with infantile Pompe disease receiving ERT for further effects of cardiac remodeling and to determine whether the immediate or long-term risk of tachyarrhythmias and sudden deaths changes with therapy. Future monitoring may also include heart rate variability, which in adults has been associated with congestive heart failure.

\section{ACKNOWLEDGMENTS}

We thank Joanne Mackey, PNP, and Stephanie DeArmey, PA, for the excellent clinical care of these critically ill babies.

\section{References}

1. Martiniuk F, Chen A, Mack A, Arvanitopoulos E, et al. Carrier frequency for glycogen storage disease type II in New York and estimates of affected individuals born with the disease. Am J Med Genet 1998;79:69-72.

2. Hirschhorn R, Reuser AJ. Glycogen storage disease type II: acid alpha-glucosidase (acid maltase) deficiency. In: The Metabolic and Molecular Bases of Inherited Disease. Scriver C, Beaudet A, Sly W, Valle D, Childs B, et al. (eds.). New York: McGraw Hill, 2001:3389-3420.

3. Kishnani P, Wuh-Liang Hwu, Mandel H, Nicolino M, et al. A retrospective, multinational, multicenter study on the natural history of infantile-onset Pompe disease. J Pediatr (in press).

4. Kishnani PS, Howell RR. Pompe disease in infants and children. J Pediatr 2004;144: S35-S43.

5. Bulkley BH, Hutchins GM. Pompe's disease presenting as hypertrophic myocardiopathy with Wolff-Parkinson-White syndrome. Am Heart J 1978;96:246-252. 
6. Francesconi M, Auff E, Ursin C, Sluga E. [WPW syndrome combined with AV block 2 in an adult with glycogenosis (Type II)]. Wien Klin Wochenschr 1982;94: 401-404.

7. Tabarki B, Mahdhaoui A, Yacoub M, Selmi H, et al. [Familial hypertrophic cardiomyopathy associated with Wolff-Parkinson-White syndrome revealing type II glycogenosis]. Arch Pediatr 2002;9:697-700.

8. Klinge L, Straub V, Neudorf U, Schaper J, et al. Safety and efficacy of recombinant acid alpha-glucosidase (rhGAA) in patients with classical infantile Pompe disease: results of a phase II clinical trial. Neuromuscul Disord 2005;15:24-31.

9. Klinge L, Straub V, Neudorf U, Voit T. Enzyme replacement therapy in classical infantile pompe disease: results of a ten-month follow-up study. Neuropediatrics 2005;36:6-11.

10. Kishnani PVT, Nicolino M, Tsai C-H, Herman G, et al. Enzyme replacement therapy with recombinant human acid alpha glucosidase (rhGAA) in infantile Pomp disease (IPD): results from a phase 2 study. Pediatr Res 2003;53:259.

11. Amalfitano A, Bengur AR, Morse RP, Majure JM, et al. Recombinant human acid alpha-glucosidase enzyme therapy for infantile glycogen storage disease type II: results of a phase I/II clinical trial. Genet Med 2001;3:132-138.

12. Van den Hout JM, Reuser AJ, de Klerk JB, Arts WF, et al. Enzyme therapy for Pompe disease with recombinant human alpha-glucosidase from rabbit milk. J Inherit Metab Dis 2001;24:266-274.
13. Ansong A, Li J, Nozik-Grayck E, Ing R, et al. Electrocardiographic response to enzyme replacement therapy for Pompe disease. Genet Med 2006;8:297-301.

14. Vogel MSW, Buhlmeyer K. Left ventricular myocardial mass determined by crosssectional echocardiography in normal newborns, infants and children. Pediatr Cardiol 1991;12:143-149.

15. Reichek N, Helak J, Plappert T, Sutton MS, et al. Anatomic validation of left ven tricular mass estimates from clinical two-dimensional echocardiography: initial results. Circulation 1983;67:348-352.

16. Schiller NB, Shah PM, Crawford M, DeMaria A, et al. Recommendations for quantitation of the left ventricle by two-dimensional echocardiography. American Society of Echocardiography Committee on Standards, Subcommittee on Quantitation of Two-Dimensional Echocardiograms. J Am Soc Echocardiogr 1989;2:358-367.

17. van den Hout HM, Hop W, van Diggelen OP, Smeitink JA, et al. The natural course of infantile Pompe's disease: 20 original cases compared with 133 cases from the literature. Pediatrics 2003;112:332-340.

18. Arad M, Moskowitz IP, Patel VV, Ahmad F, et al. Transgenic mice overexpressing mutant PRKAG2 define the cause of Wolff-Parkinson-White syndrome in glycogen storage cardiomyopathy. Circulation 2003;107:2850-2856.

19. Mignani R, Panichi V, Giudicissi A, Taccola D, et al. Enzyme replacement therapy with agalsidase beta in kidney transplant patients with Fabry disease: a pilot study. Kidney Int 2004;65:1381-1385. 